\title{
Editorial
}

Dermatology

\section{Chronic Pruritus Management: A Plea for Improvement - Can Itch Clinics Be an Option?}

\author{
Luigi Naldi $^{a}$ Santo Raffaele Mercuri ${ }^{b}$ \\ ${ }^{a}$ Centro Studi GISED and Department of Dermatology, Ospedali Riuniti, Bergamo, and \\ ${ }^{b}$ Department of Dermatology, Ospedale San Raffaele, Università Vita-Salute, Milano, Italy
}

\section{Key Words}

Itch clinics $\cdot$ Pruritus management $\cdot$ Health care research

\section{Introduction}

People with chronic pain may be able to attend a specialist pain clinic for assessment and possible pain management, together with advice on living a fuller life in spite of pain. People with chronic itch are less liable to be referred to a specialized service since, with few exceptions, itch clinics are simply not available [1]. Is chronic itching less relevant than pain?

In this issue of the journal, Ständer et al. [2] present data on the prevalence of chronic pruritus in the German working population. The results point to a remarkably high prevalence of the symptom in the population, suggesting that effective interventions are not available or not applied. Chronic pruritus was reported by about $17 \%$ of the German sample with about $4 \%$ of all persons reporting the symptom being continuously present. About $89 \%$ of the people with chronic pruritus reported being bothered, and nearly $11 \%$ being greatly bothered, by the symptom. Interestingly, people complaining of frequent or constant pruritus had a higher intensity than those with occasional occurrence of the symptom, pointing to chronicity as affecting severity (a phenomenon similar to what has been observed in people with chronic pain) $[3,4]$. About half of the people suffering from pruritus had never sought medical advice for their symptom. There is an obvious need for patient education and for improvement in treatment options. The establishment of itch clinics may help.

\section{Improving Clinical Assessment}

An area in need of improvement is clinical classification and assessment. A first attempt to standardize clinical assessment has been made by the International Forum for the Study of Itch [5]. An agreement was reached on identifying 3 groups of conditions: pruritus on diseased (inflamed) skin (group I), pruritus on nondiseased (noninflamed) skin (group II) and pruritus presenting with severe chronic secondary scratch lesions, such as prurigo nodularis (group III). In addition, according to the underlying disease, different categories were considered: dermatological diseases, systemic diseases including diseases of pregnancy and drug-induced pruritus, neurological and psychiatric diseases. 'Mixed' cases, i.e. patients with $>1$ cause, and cases without any identifiable cause,

\section{KARGER}

Fax +4161306 1234

E-Mail karger@karger.ch

www.karger.com
C) 2010 S. Karger AG, Basel

$1018-8665 / 10 / 2213-0216 \$ 26.00 / 0$

Accessible online at: www.karger.com/drm
Luigi Naldi, MD

Centro Studi GISED, Fondazione per la Ricerca Ospedale Maggiore

Unità di Dermatologia, Ospedali Riuniti

Via Garibaldi 13-15, IT-24100 Bergamo (Italy)

Tel. +390352 278 719, Fax +390352 278 673, E-Mail luigi.naldi@gised.it 
Table 1. Some of the proposed treatment options for controlling cholestatic pruritus

Cholestyramine

Ursodeoxycholic acid

Rifampin

$\mu$-Opioid receptor antagonists (naltrexone, naloxone, nalmefene) Phenobarbital

Propofol

Thalidomide

Ondansetron

Dronabinol

Sertraline

Stanozolol

Butorphanol spray

Plasma resin perfusion (ion resin BR-350)

Albumin dialysis

Ultraviolet light phototherapy

Exposure to bright light from a light therapy box

were also considered. This is the first version of a clinical classification. Further improvements can be made based on physiopathogical considerations. The way the classification could improve management should be assessed in field surveys.

\section{Improving Management}

The management of chronic pruritus is a multidisciplinary task involving, among others, dermatologists, neurologists and specialized nurses. Thus far, no specific antipruritic drugs exist that equal aspirin's association with pain relief. Patient education and elimination of provocative factors is an important component of itch management. The teaching of adequate methods of interrupting the itch-scratch cycle, and stress control techniques are important components as well [6]. If properly organized, itch clinics may be best suited to harmonize these different components of itch management.

As indicated by the diversity of options for controlling pruritus in a condition such as cholestasis (table 1) [7] clinical research and outcome evaluation are in an urgent need of improvement. An example of the way management can take advantages of improvements in the understanding of pathophysiology and multidisciplinary collaboration is offered by polycythemia vera. Disabling itching is seen in approximately $40 \%$ of the patients suffering from polycythemia vera and the symptom is characteristically triggered by contact with water (aquagenic)

Itch Clinics: An Option for Chronic

Pruritus Management? at any temperature. Many different treatment options have been tried over the past several decades, e.g. antihistamines, antidepressants, interferon- $\alpha$, phlebotomy, phototherapy, iron supplements and myelosuppressive medications, all demonstrating mixed results [8]. Together with essential thrombocytosis and primary myelofibrosis, polycythemia vera is a myeloproliferative clonal disorder arising in a pluripotent hematopoietic stem cell. The discovery of an activating mutation (V617F) in the gene for Janus kinase 2, a tyrosine kinase utilized by hematopoietic cell receptors for growth factors, provided an explanation for the shared clinical features of the 3 myeloproliferative disorders and offered a new potential target for treatment [9]. Preliminary results of clinical trials with agents that inhibit the mutated kinase have shown impressive clinical benefit on itching in polycitemia vera [10].

Recent research on itching has revealed new neuronal mechanisms in the skin and brain, suggesting novel therapeutic targets [11]. Specific agonists such as cannabinoids or calcineurin inhibitors can influence neuroreceptors on sensory nerve fibers of the skin, itch-selective neurons in the dorsal horn of the spinal cord can be targeted to inhibit the transmission of pruritus to the somatosensory cortex, and anticonvulsants, antidepressants and microopioid receptor antagonists may interfere with the sensation of pruritus in the central nervous system. Placebo responses in pruritus are quite marked and many systemic drugs with antipruritic effects may work primarily by a placebo mechanism, demonstrating the powerful central nervous modulation of pruritus [12]. Pruritus may be precipitated, prolonged or enhanced by a number of stress-related mediators such as histamine and neuropeptides. There are also a number of secondary psychosomatic mechanisms through which pruritus may be generated or exacerbated, e.g. sweat response, alterations in cutaneous blood flow and scratching. Limited data point to group psychotherapy, behavioral therapy, controlled physical exercise, support groups and biofeedback as effective means to stop scratching and improve quality of life. Itch clinics may assess in a formal way the impact of these interventions as part of a harmonized multicomponent management strategy [13].

\section{Organization Issues}

As discussed above, itch clinics may offer a multidisciplinary setting for the optimal management of chronic pruritus. In principle, they may also concentrate exper- 
tise and knowledge in a single spot, which may help foster research on pathomechanisms and treatment. As for any change in the organization of medical care, there is a need for documenting the advantage of specialized itch clinics in terms of improved outcome and cost-effectiveness.
This means that itch clinics should be implemented in an experimental way in limited areas to assess, in the framework of so-called 'health service research', their impact and sustainability [14]. All in all, it is worthy of consideration for improving patient care.

\section{References}

- 1 Van Os-Medendorp H, Ros WJ, Elandde Kok PC, Kennedy C, Thio BH, van der Schuur-van der Zande A, Grypdonck $\mathrm{MH}$, Bruijnzeel-Koomen CA: Effectiveness of the nursing programme 'Coping with itch': a randomized controlled study in adults with chronic pruritic skin disease. Br J Dermatol 2007;156:1235-1244.

2 Ständer S, Schäfer I, Phan NQ, Blome C, Herberger $\mathrm{K}$, et al: Prevalence of chronic pruritus in Germany - results of a cross-sectional study in a sample working population of 11730. Dermatology DOI: $\underline{10.1159 /}$ 000319862 .

$>3$ Turk DC, Wilson HD: Fear of pain as a prognostic factor in chronic pain: conceptual models, assessment, and treatment implications. Curr Pain Headache Rep 2010;14:8895.

4 Dalgard F, Dawn AG, Yosipovitch G: Are itch and chronic pain associated in adults? Results of a large population survey in Norway. Dermatology 2007;214:305-309.
5 Ständer S, Weisshaar E, Mettang T, Szepietowski JC, Carstens E, Ikoma A, Bergasa NV, Gieler U, Misery L, Wallengren J, Darsow U, Streit M, Metze D, Luger TA, Greaves MW, Schmelz M, Yosipovitch G, Bernhard JD: Clinical classification of itch: a position paper of the International Forum for the Study of Itch. Acta Derm Venereol 2007;87:291294.

6 Misery L: Are Pruritus and scratching the cough of the Skin? Dermatology 2008;216: 3-5.

$>7$ Zein CO, Lindor KD: Latest and emerging therapies for primary biliary cirrhosis and primary sclerosing cholangitis. Curr Gastroenterol Rep 2010;12:13-22.

8 Saini KS, Patnaik MM, Tefferi A: Polycythemia vera-associated pruritus and its management. Eur J Clin Invest 2010;40:828-834.

$\checkmark 9$ Tefferi A: JAK2 mutations in polycythemia vera: molecular mechanisms and clinical applications. N Engl J Med 2007;356:444445 .
10 Spivak JL: Narrative review: thrombocytosis, polycythemia vera, and JAK2 mutations: the phenotypic mimicry of chronic myeloproliferation. Ann Intern Med 2010;152: 300-306.

11 Pogatzki-Zahn E, Marziniak M, Schneider G, Luger TA, Ständer S: Chronic pruritus: targets, mechanisms and future therapies. Drug News Perspect 2008;21:541-551.

12 McGlone F, Reilly D: The cutaneous sensory system. Neurosci Biobehav Rev 2010;34:148159.

13 Bathe A, Matterne U, Dewald M, Grande T, Weisshaar E: Educational multidisciplinary training programme for patients with chronic pruritus. Acta Derm Venereol 2009;89: 498-501.

14 Hussey PS, de Vries H, Romley J, Wang MC, Chen SS, Shekelle PG, McGlynn EA: A systematic review of health care efficiency measures. Health Serv Res 2009;44:784-805. 